\title{
Differences between models of partial thickness and subendocardial ischaemia in terms of sensitivity analyses of ST-segment epicardial potential distributions
}

\author{
Barbara M Johnston $^{\mathrm{a}, *}$, Peter R Johnston ${ }^{\mathrm{a}}$ \\ ${ }^{a}$ School of Environment and Science, and Queensland Micro- and Nanotechnology Centre, Griffith University, Nathan, Queensland, \\ Australia, 4111
}

\begin{abstract}
Mathematical modelling is a useful technique to help elucidate the connection between non-transmural ischaemia and ST elevation and depression of the ECG. Generally, models represent non-transmural ischaemia using an ischaemic zone that extends from the endocardium partway to the epicardium. However, recent experimental work has suggested that ischaemia typically arises within the heart wall. This work examines the effect of modelling cardiac ischaemia in the left ventricle using two different models: subendocardial ischaemia and partial thickness ischaemia, representing the first and second scenarios, respectively.

We found that it is possible, only in the model of subendocardial ischaemia, to see a single minimum on the epicardial surface above the ischaemic region, and this only occurs for low ischaemic thicknesses. This may help to explain the rarity of ST depression that is located over the ischaemic region. It was also found that, in both models, the epicardial potential distribution is most sensitive to the proximity of the ischaemic region to the epicardium, rather than to the thickness of the ischaemic region. Since proximity does not indicate the thickness of the ischaemic region, this suggests a reason why it may be difficult to determine the degree of ischaemia using the ST segment of the ECG.
\end{abstract}

Keywords: Partial thickness ischaemia; Subendocardial ischaemia; ST depression; Sensitivity analysis; Bidomain model; Gaussian Process emulators

\section{Introduction}

Mathematical modelling of cardiac electrophysiology provides a useful tool to improve our understanding of cardiac diseases and to simulate and test processes that are difficult to perform experimentally [1]. In the case of myocardial ischaemia, which arises from a reduced blood supply to the heart, mathematical models have the potential to shed light on the difficulties associated with the detection of non-transmural ischaemia via the electrocardiogram (ECG) $[2,3,4,5]$.

This is an on-going area of research, since it is desired, but not yet possible, to use ECG recordings in a satisfactory manner to detect and localise non-transmural ischaemia [6]. At present, clinicians are able to use elevations in the ST-segment to detect and localise transmural ischaemia, but the same is not true for non-transmural ischaemia, where the method results in unsatisfactory sensitivity and specificity values [7].

Another aspect of this research is the search for a more fundamental understanding of how and where myocardial ischaemia arises. Opposing viewpoints suggest, on the one hand, that ischaemia initially develops from the endocardium towards the epicardium (subendocardial ischaemia) $[8,9,10]$, and on the other hand, that is possible that it

${ }^{*}$ Corresponding author is more likely to initially develop in the mid-wall (partial thickness ischaemia) [11].

A recent experimental study has leant weight to the latter point of view, since it was found that acute ischaemia arose throughout the myocardium, and not solely in the subendocardium, in $87 \%$ (canine) and $94 \%$ (swine) of episodes studied [11]. The study also found that ischaemia did not occur in a single region, but developed in a number of smaller regions throughout the mid-myocardium [11], which then joined together as ischaemic progressed and spread transmurally to the epicardium, as found in previous studies $[8,9]$. To date, most mathematical models simply represent non-transmural ischaemia as a single (sometimes variously shaped) subendocardial zone $[4,5$, $12,13,14]$.

A recent study [15] has attempted to shed light on the above, by modelling acute ischaemia during the STsegment of the ECG, using subject-specific ischaemic zones, and comparing the results with measured epicardial potentials. The study found that zone-based methods produced potentials that matched measured epicardial potentials more effectively than those produced using needle measured intracardiac potentials as sources, and that care needed to be taken with the choice of the border-zone parameters.

This concern with the choice of model parameters is an example of a wider concern associated with mathematical 
modelling, in general, and cardiac modelling, in particular; that is, the inputs to these types of models are often difficult to measure (e.g. the cardiac conductivity values) or they can vary across populations, with possible effects on the accuracy of the outputs that are produced [1].

A number of cardiac modelling studies that quantify the effect of this uncertainty or look at the sensitivity of the outputs to uncertainty in the inputs have been performed in recent years. Techniques used in such studies include a population of models approach [16], the use of generalised polynomial chaos $[17,18,19]$ and Monte-Carlo approaches $[20,21]$.

Another approach is to use Gaussian Process emulators $[22,23]$, which connect the inputs to the outputs via a fast-running surrogate model. This has the advantage of drastically reducing the number of model runs that would be required with a Monte-Carlo based method. Studies in cardiac electrophysiology that use Gaussian Process emulators have been conducted primarily in cell models $[21,23,24]$, but more recently there have been some in tissue level models $[13,20,25,26]$.

This study investigates the similarities and differences between two different ST-segment bidomain models of ischaemia, subendocardial and partial thickness ischaemia, in an ellipsoid-shaped ventricle and also in a more realistic heart. We do not consider ischaemia that extends to full-thickness since our aim is to look at the connection between acute ischaemia, epicardial potentials and their possible connection to ST depression in the ECG. We simultaneously consider the effect of different choices for representing the start of the ischaemic region, as well as the effects of representations for Early stage and Late stage ischaemia, on the form of the epicardial potential distributions (EPDs) that are produced.

In the half-ellipsoidal model, we make these comparisons by using Gaussian process emulators to systematically investigate the sensitivity of the features of the EPDs (the outputs) to the various inputs of the models, as well as to quantify the effects of uncertainty in the inputs.

Our previous work used a subendocardial model of ischaemia [27] primarily to investigate and quantify the effect of reducing the conductivity values in the ischaemic region to model Early and Late stages of ischaemia. As in previous studies $[28,4,5]$, we found that three types of EPDs can be identified, depending primarily on how deep the ischaemic region extends into the ventricular wall from the endocardium. We have named these EPDs as: type 1 (a single minimum over the ischaemic region); type 2 (a maximum over the ischaemic region, flanked by a single minimum), and type 3 (like type 2 , but flanked by a second minimum). Also as in previous studies [29, 30], we found that the positions of some of these extrema are affected by the degree of rotation of the sheets of cardiac fibres between the epicardium and the endocardium. One other major finding [27] was that the blood conductivity had no effect on the EPDs and that the effect of the six bidomain conductivity values was minor, in comparison with the ef- fect of ischaemic depth. This work will investigate whether these conclusions still hold when modelling partial thickness ischaemia, rather than subendocardial ischaemia.

\section{Methods}

\subsection{Governing Equations}

This work compares simulations using two different models of ischaemia in the left ventricle: partial thickness ischaemia (PTI) and subendocardial ischaemia (SI). The methods used here for the PTI model parallel those used in our previous SI study [27] to allow for comparisons to be made between the two studies. Some differences in methods do arise, however, because a new parameter is necessary to indicate the starting position of the ischaemic region in the case of the PTI model.

In both cases, the ventricular muscle is approximated by a half-ellipsoid (Figure 1), which is assumed to be filled with blood and is open to the air.

The equations for the half-ellipsoid are

$$
x=a \cos \theta \cos \phi, \quad y=a \cos \theta \sin \phi, \quad z=c \sin \theta
$$

where $0 \leq \theta \leq \pi / 2,-\pi \leq \phi \leq \pi$, and the endocardial surface corresponds to $a=2 \mathrm{~cm}, c=4 \mathrm{~cm}$, while $a=3$ $\mathrm{cm}, c=5 \mathrm{~cm}$ gives the epicardial surface.

In the SI case, the ischaemic region begins at the endocardium and extends partway into the tissue for a distance referred to as the ischaemic depth (ISC), which is given as a percentage of the total wall thickness (Figure 1(a), where the subendocardial region is marked with the letter ' $\mathrm{S}$ '). A few SI simulation results are presented here as a comparison to the PTI simulations, but the full details and analysis of those simulations can be found in our previous study [27].

The difference in the PTI case is that the starting position for the ischaemic tissue is not at the endocardium, but further into the ventricular wall, as shown in Figure 1(b), where the partial thickness ischaemic region is marked with the letter ' $\mathrm{P}$ '. In both models, the ischaemic region is approximately rectangular in shape and of size $\left(50^{\circ} \leq \theta \leq 70^{\circ},-15^{\circ} \leq \phi \leq 15^{\circ}\right.$ and depth ISC $)$.

The effects of the intracellular and extracellular regions of the ventricular tissue are taken into account by the use of the bidomain model for cardiac tissue [31, 32], which considers that the electrical behaviour of the tissue occurs in two interpenetrating domains, intracellular $(i)$ and extracellular $(e)$. Due to the anisotropic nature of the tissue, the electrical conductivity is different in each of three orthogonal directions in the model: longitudinal $(l)$; transverse $(t)$, and normal $(n)$, where the first two are within a sheet of cardiac fibres, with $l$ along the fibres, $t$ orthogonal to this, and $n$ normal to the sheets. Thus the bidomain model requires six conductivities $\left(g_{p q}, p=i, e, q=l, t, n\right)$, 


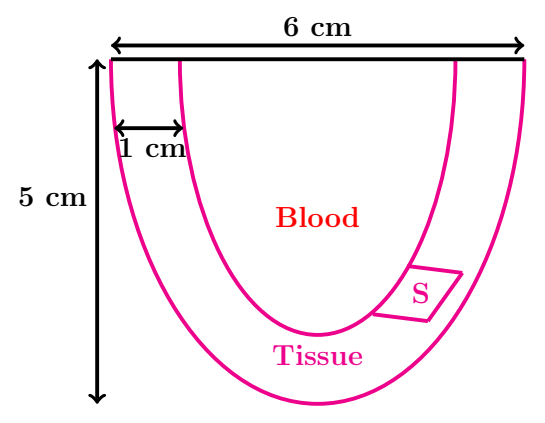

(a) SI model

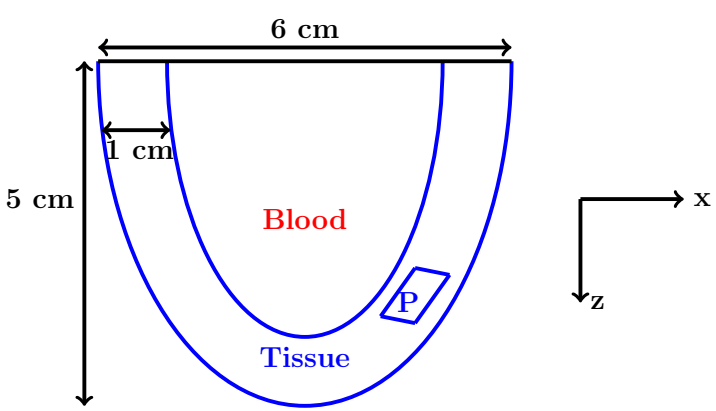

(b) PTI model

Figure 1: Cross-sectional view of the $x-z$ plane at $y=0$, for the half-ellipsoidal model. The subendocardial ischaemic region is marked with the letter $\mathrm{S}$ in (a) and the partial thickness ischaemic region is marked with the letter $\mathrm{P}$ in (b).

where the subscripts represent first the domain and second the direction of conduction.

In this work, the passive bidomain equation [4] connects the intracellular and extracellular potentials, $\phi_{i}$ and $\phi_{e}$, respectively, via the transmembrane potential $\phi_{m}=$ $\phi_{i}-\phi_{e}$, because the simulation is conducted during the iso-electric ST-segment of the ECG. We assume that $\phi_{m}$ is a known function of position. Thus the governing equations are

$$
\nabla \cdot\left(\mathbf{M}_{i}+\mathbf{M}_{e}\right) \nabla \phi_{e}=-\nabla \cdot \mathbf{M}_{i} \nabla \phi_{m}
$$

and

$$
g_{b} \nabla^{2} \phi_{b}=0
$$

where $\phi_{b}$ is the potential in the blood and $g_{b}$ is the blood conductivity. The conductivity tensors $\mathbf{M}_{p}=\mathbf{A} \mathbf{G}_{p} \mathbf{A}^{T}(p=$ $i, e)$ incorporate the fibre rotation matrix $\mathbf{A}$ and the conductivity values $\left(g_{p q}, q=l, t, n\right)$, which are contained in the diagonal matrix $\mathbf{G}_{p}$. We assume the fibre rotation varies linearly from epicardium (where the cardiac fibres are offset by $-45^{\circ}[33]$ ) to the endocardium, with a total fibre rotation angle (ROT), given in degrees, and that the sheets of fibres remain parallel to the epicardium [34].

We represent $\phi_{m}$ by the expression

$$
\phi_{m}(r, \theta, \phi)=\Delta \phi_{p} \Psi_{c}\left(r_{a}-r\right) \Psi_{c}\left(\theta-\theta_{0}\right) \Psi_{c}(\phi)
$$

where $\Delta \phi_{p}$ is the difference between the normal and ischaemic tissue plateau potentials, and is set to $-30 \mathrm{mV}$ $[4,33]$. The two representations of the transmembrane potential $\Psi_{c}$ are discussed below.

If we centre the ischaemic region at $\left(r_{a}, \theta_{0}, 0\right)$, then the first representation for $\Psi_{c}, \Psi_{1}(t)$, is given by

$$
\Psi_{1}(t)= \begin{cases}\frac{1-\exp \left(-a_{t} / \lambda_{t}\right) \cosh \left(t / \lambda_{t}\right)}{1-\exp \left(-a_{t} / \lambda_{t}\right)} & |t| \leq a_{t} \\ \frac{\exp \left(-|t| / \lambda_{t}\right) \sinh \left(a_{t} / \lambda_{t}\right)}{1-\exp \left(-a_{t} / \lambda_{t}\right)} & |t|>a_{t}\end{cases}
$$

where $a_{t}$ is the half-width of the ischaemic region, for $t=r, \theta, \phi$. We use a narrow border region between the ischaemic zone and the normal tissue [25, 27] by setting $\lambda_{t}=0.01$ in each of the above directions (Figure 2).

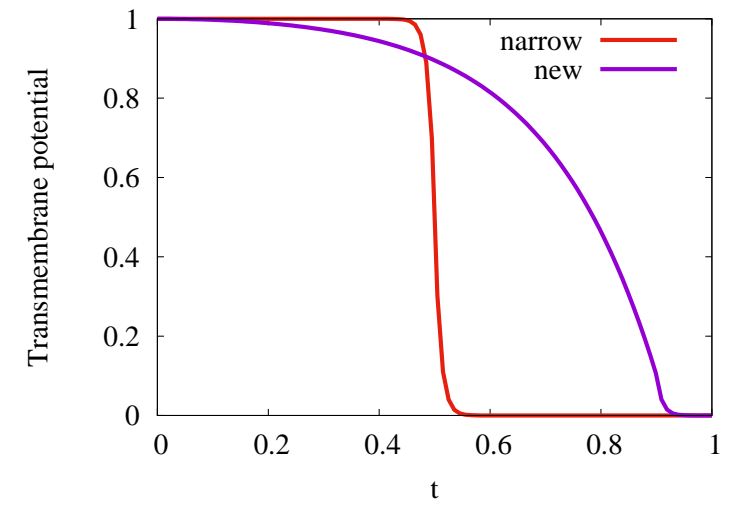

Figure 2: Transmembrane potentials for the narrow border zone and the new representation (equation (6)).

As a comparison, we consider (Figure 2) a wider and slightly differently shaped (which is referred to as 'new') version of $\Psi_{c}, \Psi_{2}[25,27]$, which more closely matches experimental results $[5,35,36]$, given by

$$
\Psi_{2}(t)= \begin{cases}\frac{1-2\left(1-a_{2}\right) \exp \left(-a_{1} / b_{1}\right) \cosh \left(t / b_{1}\right)}{1-2\left(1-a_{2}\right) \exp \left(-a_{1} / b_{1}\right)} & |t| \leq a_{1} \\ \frac{2 a_{2} \exp \left(-|t| / b_{2}\right) \sinh \left(a_{1} / b_{2}\right)}{1-2\left(1-a_{2}\right) \exp \left(-a_{1} / b_{2}\right)} & |t|>a_{1}\end{cases}
$$

with $a_{1}=0.9, b_{1}=0.2, b_{2}=0.01, a_{2}=0.1$. Here $\Psi_{2}$ is used in the $t=\theta$ and $t=\phi$ directions, with $\Psi_{1}$ in the $t=r$ direction.

As in our earlier work [25, 27], the model is solved using techniques based on the finite volume method, which have been validated previously [33]. The mesh for the half-ellipsoidal model is constructed from 423,975 nodes and 414,832 hexahedral elements [33]. The boundary conditions that are applied are: that there is no current flux at the intracellular or extracellular domain boundaries; or at the blood or tissue interfaces with the air; and that there is continuity of potential and extracellular current at the blood tissue interface [33]. Details of the solution technique can be found in [33]. 


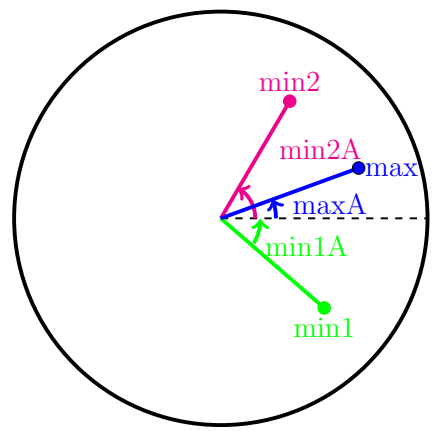

Figure 3: The angles min1A, maxA and min2A are illustrated for an example of an EPD polar plot with features min1, max, min2.

\subsection{Parameter values}

We solve the half-ellipsoid model initially using mean parameter values as inputs [25], as listed in Table 1, for ROT, $g_{b}$ and $g_{p q}(p=i, e, q=l, t, n)$. Depending on whether we are simulating Early or Late stage ischaemia, we reduce the conductivities in the ischaemic region by the fractional reductions $\left(f_{p q}\right.$, Table 1$)$, which operate on the corresponding value of $g_{p q}$ in the ischaemic region only. These values have been used in our previous work [27] and are those used by Hopenfeld et al. [4]. The $f_{p q}$ values reflect the fact that only the extracellular conductivities are affected in early (acute) stage ischaemia, whereas there is a steep drop in the intracellular conductivities during the later stages of ischaemia $[29,37]$.

\section{3. $E P D s$}

Using the above-mentioned parameters and solution technique, we solve the half-ellipsoid model with PTI and produce EPDs. We present the EPDs as polar plots, which are created by flattening the epicardial ellipsoidal surface to make a circle, with the apex at the centre $\left(\phi_{e}\right.$ is set to zero there). Each point $(r, \theta, \phi)$ on the ellipsoid is mapped onto a point on the circle, whose radius is determined by its value of $\theta$ and whose angle is determined by its value of $\phi$.

The main features of the EPDs that we are interested in are the extrema and their positions. An example of an EPD with one maximum (marked 'max') and two minima (marked 'min1' and 'min2') is given in Figure 3. The corresponding angles for these features, maxA, min1A and $\min 2 \mathrm{~A}$, respectively, are also shown in the figure.

In addition to producing mean EPDs (that is, those found using mean parameter values from Table 1), we also use ranges of these parameters to determine the sensitivity of these EPD features to changes in the input parameters. Two methods, Gaussian Process (GP) emulators and Partial Least Squares (PLS) regression are used for this analysis.
Table 1: Mean parameter values used in the Early and Late stage ischaemia simulations. The parameters $f_{p q}$ represent fractional reductions (in the ischaemic region) in the corresponding bidomain conductivities $g_{p q},(p=i, e, q=l, t, n), g_{b}$ is the blood conductivity and ROT is the fibre rotation angle. Conductivities are in $\mathrm{mS} / \mathrm{cm}$.

\begin{tabular}{l|l}
\hline Stage & Parameter values \\
\hline Early & $f_{\text {el }}=0.5, f_{\text {et }}=0.25, f_{\text {en }}=0.25$ \\
& $f_{i l}=0, f_{i t}=0, f_{\text {in }}=0$ \\
\hline Late & $f_{e l}=0.5, f_{\text {et }}=0.25, f_{\text {en }}=0.25$ \\
& $f_{i l}=0.9, f_{i t}=0.98, f_{\text {in }}=0.98$ \\
\hline Both & $\mathrm{ROT}=100^{\circ}, g_{b}=6.5$, \\
& $g_{e l}=2.4, g_{\text {et }}=1.6, g_{\text {en }}=1.0$ \\
& $g_{i l}=2.4, g_{i t}=0.24, g_{i n}=0.1$ \\
\hline
\end{tabular}
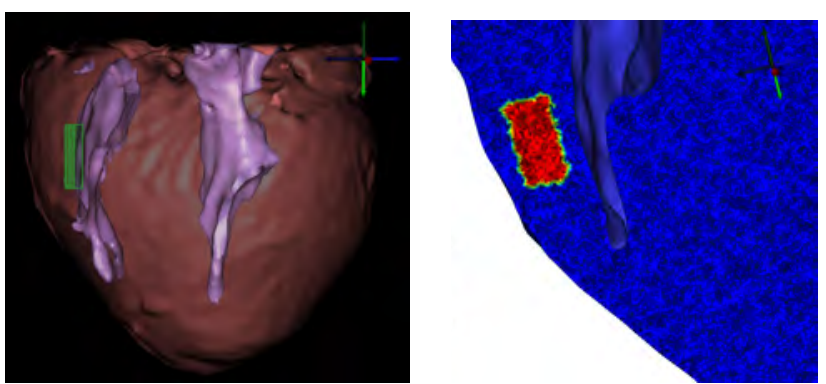

Figure 4: The realistic heart with (a) a subendocardial ischaemic region and (b) the mesh with a partial thickness ischaemic region.

\subsection{Realistic model}

As a comparison and check, we also produce mean (Table 1) simulations in a (more) realistic whole heart canine geometry (Figure 4(a)), which has blood-filled ventricles that are open to the air and includes a septum. A similar rectangularly-shaped ischaemic patch to that used in the half-ellipsoidal model, is used in this model (Figure 4(b)). The mesh for the realistic model uses 717,709 nodes and 4,486,917 tetrahedral elements [38]. The realistic model is solved with a previously validated technique based on the finite volume method. Details are given in [25, 33, 38].

\subsection{EPD analysis methods}

For both methods, we first create sets of design data by varying the mean inputs in Table 1 (excluding the $f_{p q}$ values) across the ranges mean $\pm 50 \%$, using a Latin Hypercube sampling routine. These sets of data are then used as inputs to the PTI model, which produces an EPD for each set of inputs. From these EPDs, we extract outputs associated with the features, such as min1A, maxA and min2A (Figure 3), and their corresponding potentials $\min 1 \mathrm{~V}, \max \mathrm{V}$ and $\min 2 \mathrm{~V}$, respectively.

\subsubsection{Partial Least Squares Regression}

With the PLS method, a set of components is found from the design data that simultaneously decomposes both the input and output vectors [39, 40]. The method works 
along the same lines as principal component analysis, but it chooses the components to maximise the covariance between the outputs and the inputs. It produces a set of signed regression coefficients using the Nonlinear Iterative Partial Least Squares algorithm [39, 41], and these coefficients indicate the inputs to which the outputs are most sensitive, as well as whether an increase in an output will lead to an increase (positive coefficient) or a decrease (negative coefficient) in the output.

\subsubsection{Gaussian Process Emulator}

An alternative approach, to producing sensitivity values such as those found by the PLS method, is to first use the design data to construct a GP emulator [23]. This can then be used to perform both uncertainty quantification and sensitivity analysis. The software used, both for this and the Latin Hypercube sampling, is the package GP_emu_UQSA (DOI 10.5281/zenodo.215521). This software fits an emulator for each output, initially using a training subset of around $90 \%$ of the design data. The emulator is fitted using a Gaussian covariance function and a linear mean, and, after checking the emulator for accuracy against the remaining $10 \%$ of the data, a final emulator is built using the combined datasets [23].

The software can then be used to produce main effect plots, which show the relationship between the inputs $\mathbf{x}$ and the mean effect for a particular output $y=f(\mathbf{x})$. The definition of mean effect is that it is the conditional expectation of an output, after averaging over the remaining inputs, conditional on the input variable $x_{w}$. This is calculated for a particular output, for each of the inputs $x_{w}$, by allowing $x_{w}$ to vary over $[0,1]$, while taking the other inputs to be independently normally distributed with a mean of 0.5 and a variance of 0.04 [23].

In addition to these main effect plots, we also calculated main effect sensitivity indices [23], so as to quantify the contribution of each of the inputs to an output. The sensitivity index is unsigned because it is found as the ratio of the variance (Var) of the mean effect to the variance of the model output, for each $x_{w}$; that is,

$$
\text { Sensitivity }=\frac{\operatorname{Var}\left[E\left\{f(x) \mid x_{w}\right\}\right]}{\operatorname{Var}\{f(x)\}} .
$$

\section{Results}

\subsection{Mean EPDs for the half-ellipsoidal model}

Our previous work with the SI model [25, 27] has indicated that ISC is one of the main contributors to changes in the EPDs produced by the simulations. In the case of modelling PTI, we need to consider not only ISC but the position at which the ischaemic region begins (ISCON) and the position at which the ischaemic region ends (ISCOFF), bearing in mind that they are related by the expression ISC $=$ ISCOFF-ISCON. Both ISCON and ISCOFF will be given as $\%$ depths of the ventricular wall, where $0 \%$ corresponds to the endocardium.
Table 2: Potentials (in $\mathrm{mV}$ ) for various features of EPDs for Early stage ischaemia (Table 1) with $\mathrm{ISC}=20 \%$, for the half-ellipsoidal model. Values above the line are for the PTI model, and below the line for the SI model.

\begin{tabular}{c|ccc}
\hline ISCON-ISCOFF & $\min 1 \mathrm{~V}$ & $\operatorname{maxV}$ & $\min 2 \mathrm{~V}$ \\
\hline $10-30$ & -0.12 & 0.57 & -0.02 \\
$20-40$ & -0.12 & 0.76 & -0.08 \\
$30-50$ & -0.15 & 1.02 & -0.23 \\
$40-60$ & -0.19 & 1.37 & -0.45 \\
$50-70$ & -0.27 & 1.84 & -0.81 \\
$60-80$ & -0.39 & 2.50 & -1.43 \\
$70-90$ & -0.63 & 3.63 & -2.67 \\
\hline $0-20$ & -0.52 & 0.07 & -
\end{tabular}

In the following Sections 3.1.1-3.1.4, we will investigate the way in which the form of the EPDs is influenced by changes in the above three depth-related parameters, by considering mean EPDs.

\subsubsection{Early stage ischaemia with fixed ISC}

We begin by simulating Early stage ischaemia (Table 1) with the PTI model and fixing $\mathrm{ISC}=20 \%$, while varying ISCON from 10-70\% (and hence ISCOFF from 30-90\%). In addition, a plot for the SI model with $\mathrm{ISC}=20 \%$ is included for comparison purposes. The resulting EPDs are presented in Figure 5 and the corresponding potentials for $\min 1 \mathrm{~V}, \max \mathrm{V}$ and $\min 2 \mathrm{~V}$, are given in Table 2.

One immediate observation that can be made from Figure 5 is that all the plots are type 3 EPDs [25, 27]; that is, there is a maximum over the ischaemic region that is flanked by two minima. This is not the case for the SI simulation (Figure 5(h)), which does not have a second minimum (i.e. $\min 2$ ).

For the PTI simulations in Figure 5, we see that the potential for each feature appears to increase in magnitude as ISCON and ISCOFF increase, and this is confirmed by the values in Table 2 . The potentials for the features in the SI simulation with $\mathrm{ISC}=20 \%$ are quite different from those in the PTI simulations, with its value for min1V higher than most of the PTI values, but its value for maxV much lower than the PTI values.

\subsubsection{Early and Late stage ischaemia with fixed ISC}

Since Section 3.1.1 investigates EPDs for Early stage ischaemia, in this section we compare Early and Late stage ischaemia EPDs, this time for the larger, but still fixed, value of $\mathrm{ISC}=40 \%$. The plots are presented in Figure 6 and their corresponding potentials in Table 3, for ISCON from $10-40 \%$. Once again matching SI plots and potentials are presented (for ISC $=40 \%$ ).

In both the Early and Late ischaemia cases, we see that all the PTI plots are of EPD type 3, with the potentials of all three features increasing in magnitude with increasing 


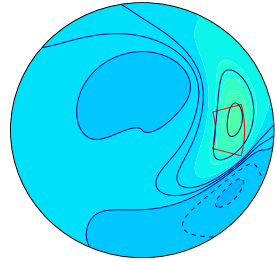

(a) $10-30 \%$

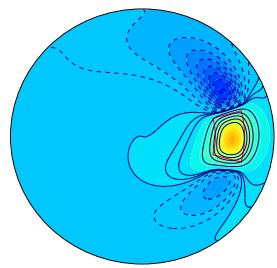

(e) $50-70 \%$

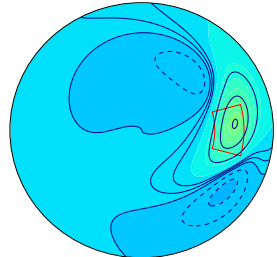

(b) $20-40 \%$

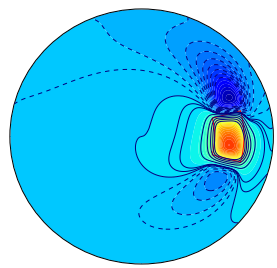

(f) $60-80 \%$

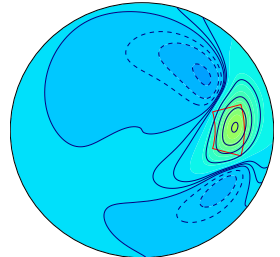

(c) $30-50 \%$

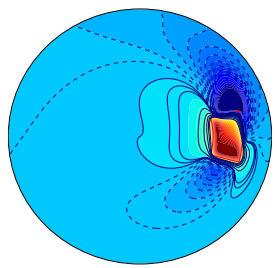

(g) $70-90 \%$

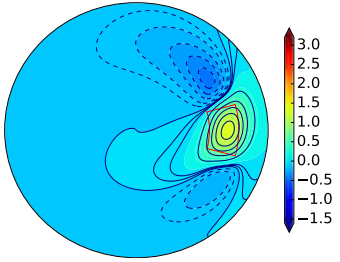

(d) $40-60 \%$

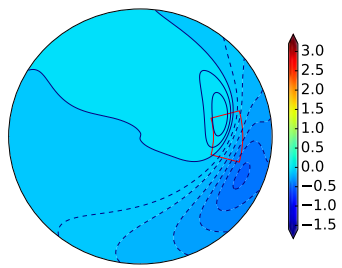

(h) $0-20 \%$

Figure 5: Epicardial potential distributions generated using mean values for the parameters (Table 1) during the Early stage of ischaemia, for a fixed ischaemic depth ISC $=20 \%$ and a range of ISCON and ISCOFF values, for the PTI and SI half-ellipsoidal models, (a)-(g) and (h), respectively. The ischaemic region is outlined in red. Dashed lines are negative potentials and solid lines zero and positive potentials. Scale is in $\mathrm{mV}$. Contours are at $-0.9,-0.8, \ldots,-0.1,-0.05,0,0.025,0.05,0.25,0.5, \ldots, 1.25 \mathrm{mV}$.

Table 3: Potentials (in $\mathrm{mV}$ ) for various features of EPDs for Early and Late stage ischaemia (Table 1) with $\mathrm{ISC}=40 \%$, for the halfellipsoidal model. Values above the line are for the PTI model, and below the line for the SI model.
Although we again have type 3 EPDs, the PTI potentials min $1 \mathrm{~V}$ and $\min 2 \mathrm{~V}$ appear to follow a trend that is opposite to the previous two studies (Sections 3.1.1 and 3.1.2); that is, their magnitudes decrease as ISCON increases. However, this is not really the case, as ISC is decreasing at the same time as (the magnitudes of) min1V and min $2 \mathrm{~V}$, so this trend could be considered to be similar to previously, if we consider ISCOFF instead of ISC. The situation for min $2 \mathrm{~V}$ is different from this, as the trend for

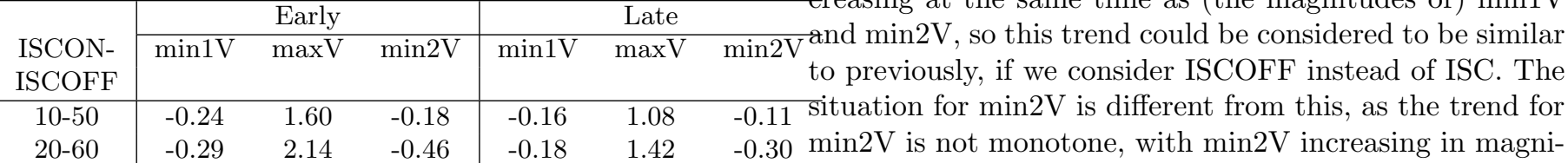

\begin{tabular}{llll|lll}
$30-70$ & -0.38 & 2.88 & -0.90 & -0.22 & 1.88 & -0.60 \\
& -0.53 & 3.87 & -1.63 & -0.30 & 2.53 & -1.09
\end{tabular}

\begin{tabular}{llll|lll}
$40-80$ & -0.53 & 3.87 & -1.63 & -0.30 & 2.53 & -1.09
\end{tabular} When we compare the PTI potentials with the values

\begin{tabular}{l|lll|lll}
$0-40$ & -0.60 & 0.76 & -0.07 & -0.36 & 0.57 & -0.03
\end{tabular} for SI simulations with ISC=70\% (Table 4), we see that the SI and PTI potentials for maxV and min2V are quite similar for low ISCON (say 10 or $20 \%$ ), but the SI min1V value has a much larger magnitude.

ISCON and ISCOFF. Although the SI plots are also of type 3 , they are of quite different magnitudes from the PTI ones, with larger magnitudes for min1V and smaller magnitudes for maxV and $\min 2 \mathrm{~V}$.

Since the trends for the PTI model appear to be similar for Early and Late stage ischaemia (albeit with lower magnitudes for Late stage ischaemia), for the remainder of the mean plots, we will concentrate on Early stage ischaemia.

\subsubsection{Early stage ischaemia with fixed ISCOFF}

In this Section, we fix ISCOFF $=70 \%$ and vary ISCON from $10-60 \%$, with the result that ISC decreases from $60-$ 10\% (see Figure 7 and Table 4). SI plots and potentials with $\mathrm{ISC}=70 \%$ are also presented for comparison.

\subsubsection{Early stage ischaemia with fixed ISCON}

We now look at one final scenario for the PTI mean EPDs; that is, we fix ISCON $=20 \%$ and vary ISCOFF (and hence ISC). In this case, there is no corresponding SI data for comparison, since all the SI simulations have ISCON $=0 \%$. The plots (Figure 8) and potentials (Table 5) once again, like Sections 3.1 .1 and 3.1.2, show type 3 EPDs where all three features increase in magnitude with ISCOFF.

Finally, there is an interesting inference that can be drawn by noticing that the min $2 \mathrm{~V}$ values in Tables 2 and 


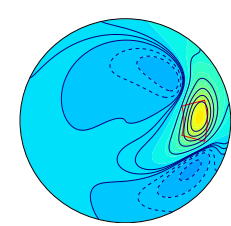

Early (a) $10-50 \%$

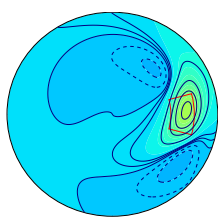

Late (a) $10-50 \%$

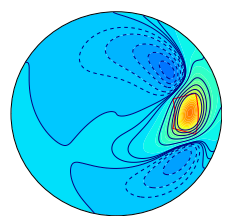

(b) $20-60 \%$

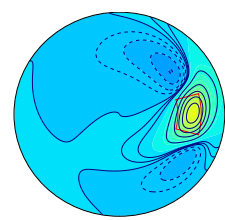

(b) $20-60 \%$

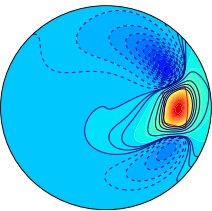

(c) $30-70 \%$

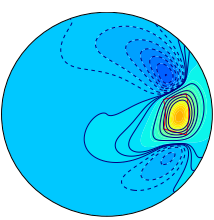

(c) $30-70 \%$

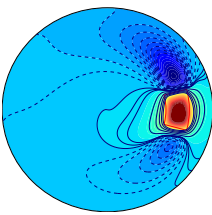

(d) $40-80 \%$

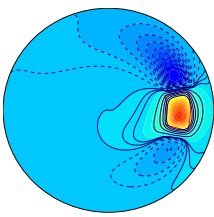

(d) $40-80 \%$

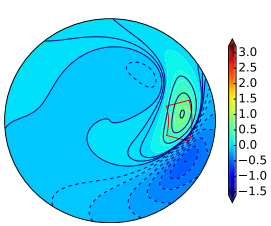

(e) $0-40 \%$

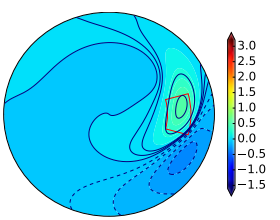

(e) $0-40 \%$

Figure 6: Epicardial potential distributions generated using mean values for the parameters (Table 1) during the Early and Late stages of ischaemia, for a fixed ischaemic depth ISC $=40 \%$ and a range of ISCON and ISCOFF values, for the PTI and SI half-ellipsoidal models, (a)-(d) and (e), respectively. See Figure 5 for contour details.

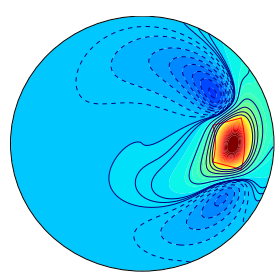

(a) $10-70 \%$

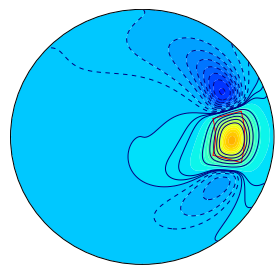

(e) $50-70 \%$

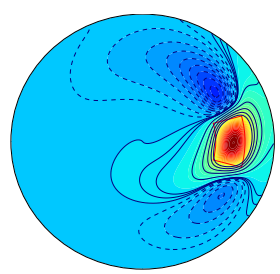

(b) $20-70 \%$

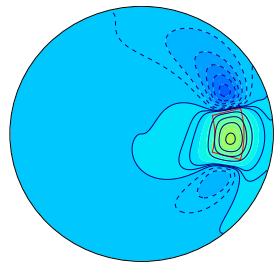

(f) $60-70 \%$

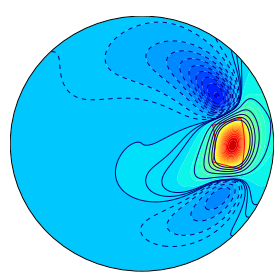

(c) $30-70 \%$

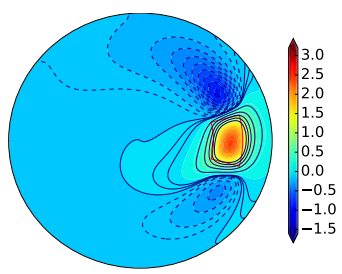

(d) $40-70 \%$

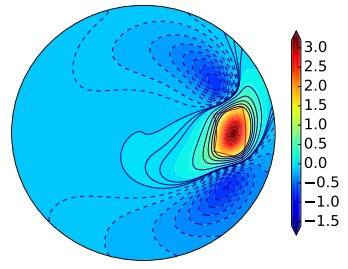

(g) $0-70 \%$

Figure 7: Epicardial potential distributions generated using mean values for the parameters (Table 1) during the Early stage of ischaemia, for a fixed ISCOFF $=70 \%$ and a range of ISCON and ISC values, for the PTI and SI half-ellipsoidal models, (a)-(f) and (g), respectively. See Figure 5 for contour details.) 
Table 4: Potentials (in $\mathrm{mV}$ ) for various features of EPDs for Early stage ischaemia (Table 1 ) with $\mathrm{ISCOFF}=70 \%$, for the half-ellipsoidal model. Values above the line are for the PTI model, and below the line for the SI model.

\begin{tabular}{c|ccc}
\hline ISCON-ISCOFF & $\min 1 \mathrm{~V}$ & $\operatorname{maxV}$ & $\min 2 \mathrm{~V}$ \\
\hline $10-70$ & -0.42 & 3.44 & -0.72 \\
$20-70$ & -0.40 & 3.20 & -0.83 \\
$30-70$ & -0.38 & 2.88 & -0.90 \\
$40-70$ & -0.34 & 2.44 & -0.92 \\
$50-70$ & -0.27 & 1.84 & -0.81 \\
$60-70$ & -0.16 & 1.04 & -0.53 \\
\hline $0-70$ & -0.81 & 3.18 & -0.77
\end{tabular}

Table 5: Potentials (in $\mathrm{mV}$ ) for various features of EPDs for Early stage ischaemia (Table 1) with ISCON $=20 \%$, for the PTI halfellipsoidal model.

\begin{tabular}{c|ccc}
\hline ISCON-ISCOFF & $\min 1 \mathrm{~V}$ & $\operatorname{maxV}$ & $\min 2 \mathrm{~V}$ \\
\hline $20-30$ & -0.06 & 0.32 & -0.02 \\
$20-40$ & -0.12 & 0.76 & -0.08 \\
$20-50$ & -0.20 & 1.35 & -0.22 \\
$20-60$ & -0.29 & 2.14 & -0.46 \\
$20-70$ & -0.40 & 3.20 & -0.83 \\
$20-80$ & -0.55 & 4.59 & -1.41 \\
$20-90$ & -0.72 & 6.47 & -2.41
\end{tabular}

5 are very similar. In Table 2 , the magnitude of min2 increases with increasing ISCOFF (with ISC fixed), while in Table 5 the magnitude of min2V increases with increasing ISCOFF (but with ISC increasing). This results in the $\min 2 \mathrm{~V}$ value for $60-80 \%$ being nearly equal to the min $2 \mathrm{~V}$ value for $20-80 \%$. In other words, min $2 \mathrm{~V}$ is not affected by the value of ISC. It is, however, sensitive to the value of ISCOFF.

In the case of $\max \mathrm{V}$ and $\min 1 \mathrm{~V}$, it is more difficult to separate the effects of ISC and ISCOFF, based on the studies undertaken so far. This is where sensitivity analysis will be a useful tool.

\subsubsection{EPDs with the new transmembrane representation}

We repeated the studies of Sections 3.1.1-3.1.4 using the new transmembrane potential representation $\Psi_{2}$ (Equation (6)) and found exactly the same trends in the EPD features as in the above sections, except that the magnitudes of the potentials were lower.

\subsection{Mean EPDs for the realistic model}

In addition, we repeated the PTI studies from Sections 3.1.1, 3.1.3 and 3.1.4, for the realistic model, to check whether they produce qualitatively similar plots and show the same trends as for the half-ellipsoidal model. Simulations that correspond to Figures 5, 7 and 8 are presented in the top two rows (labelled $\mathrm{ISC}=20 \%$ ), middle two rows (labelled $\mathrm{ISCOFF}=70 \%$ ) and bottom two rows (labelled ISCON $=20 \%$ ) of Figure 9, respectively. These plots are qualitatively similar to their counterpart half-ellipsoidal plots, and also show similar trends, and this is also true for the potential values of the features (not presented). We did not reproduce Figure 6 as it demonstrates similar trends to Figure 5, apart from the Early/Late aspect.

\subsection{Sensitivities in the half-ellipsoidal model \\ 3.3.1. PTI sensitivities of outputs to inputs for Early is- chaemia}

In order to systematically investigate the effect of the various PTI model input parameters on the six features (Figure 3) of the EPDs in the half-ellipsoidal model, we can use sensitivity analysis techniques, as described in Section 2 .

Based on our work in Section 3.1 and cognisant of the connection between ISC, ISCON and ISCOFF, we initially decided to look for sensitivity to ISC and ISCOFF, rather than to ISCON. We implemented this by first generating design data using Latin Hypercube sampling, where we varied the values of ROT and the conductivities, given in Table 1 , in the range mean $\pm 50 \%$, as described in Section 2. We did this for each of the Early and Late stage ischaemia scenarios for the PTI model, with ISCON in the range $10-30 \%$, combined with ISC in the range $5-55 \%$ (which produced ISCOFF in the range 15-85\%). This was necessary to ensure that ISCOFF was always greater than ISCON.

We found that, in each of these cases, 192 of the 200 sets of inputs produced a type 3 EPD (the other eight being type 2). In order to compare these results with SI type 3 results, we removed the eight type 2 EPDs and proceeded with the remaining 192 sets of data. We constructed GP emulators (Section 2.5.2) for each of the six outputs (the six features of the EPDs), based on the ten inputs (ROT, ISC, ISCOFF, $g_{b}$ and $\left.g_{p q}, p=i, e, q=l, t, n\right)$ for the Early Ischaemia scenario for the PTI model.

From these, we calculated sensitivity indices (Section 2.5.2) and these indices are given in Table 6 as well as PLS regression coefficients (Section 2.5.1). Also, in Table 6 are values $E^{*} E\{f(x)\}$ for the emulator expected means for each output (Section 2.5.2). Bold values indicate the inputs to which the output is particularly sensitive, as determined by both measures, for not only this study, but also similar studies which use the new transmembrane potential (Equation (6)), as well as Late ischaemia (see below). Note that the GP indices are unsigned, whereas the PLS indices are signed. Ignoring the signs, it is apparent that the two methods agree about the inputs to which the outputs are most sensitive.

As suggested in Section 3.1, we find that min2V is very sensitive to ISCOFF and not sensitive to ISC, and that 


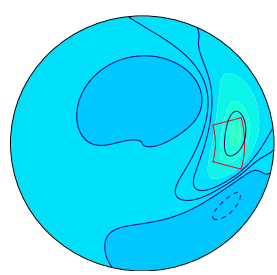

(a) $20-30 \%$

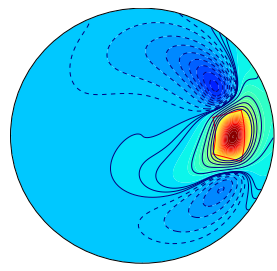

(e) $20-70 \%$

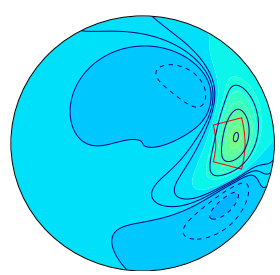

(b) $20-40 \%$

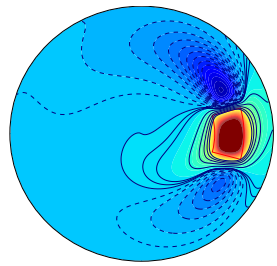

(f) $20-80 \%$

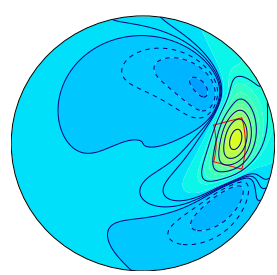

(c) $20-50 \%$

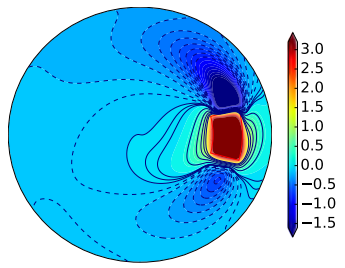

(g) $20-90 \%$

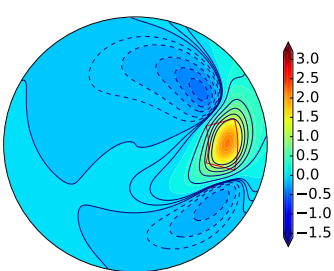

(d) $20-60 \%$

Figure 8: Epicardial potential distributions generated using mean values for the parameters (Table 1) during the Early stage of ischaemia, for a fixed ISCON $=20 \%$ and a range of ISC and ISCOFF values, for the PTI half-ellipsoidal model. (See Figure 5 for contour details.

Table 6: Emulator expected means $E^{*} E\{f(x)\}$ (in $\mathrm{mV}$ for potentials and ${ }^{\circ}$ for angles) and sensitivities of the EPD feature outputs in the PTI half-ellipsoidal model to ten inputs (see Table 1 and text), for Early stage ischaemia, calculated as GP emulator sensitivities (GP) or by partial least squares (PLS). PLS sensitivities are signed, GP sensitivities are not. Values in bold indicate those inputs to which the output is particularly sensitive.

\begin{tabular}{|c|c|c|c|c|c|c|c|c|c|c|c|c|}
\hline Feature & Method & $E^{*} E\{f(x)\}$ & ROT & ISC & ISCOFF & $g_{b}$ & $g_{i l}$ & $g_{e l}$ & $g_{i t}$ & $g_{e t}$ & $g_{\text {in }}$ & $g_{\text {en }}$ \\
\hline \multirow[t]{2}{*}{$\min 1 \mathrm{~V}$} & PLS & & -0.14 & -0.47 & -0.31 & -0.11 & -0.19 & 0.33 & 0.06 & 0.16 & 0.05 & -0.26 \\
\hline & GP & -0.22 & 0.02 & 0.19 & 0.10 & 0.01 & 0.03 & 0.08 & 0.00 & 0.02 & 0.00 & 0.05 \\
\hline \multirow[t]{2}{*}{$\min 1 \mathrm{~A}$} & PLS & & 0.67 & -0.12 & 0.24 & 0.06 & -0.20 & -0.16 & 0.00 & -0.23 & 0.04 & 0.49 \\
\hline & GP & -37.3 & 0.32 & 0.01 & 0.06 & 0.00 & 0.03 & 0.02 & 0.00 & 0.04 & 0.00 & 0.16 \\
\hline \multirow[t]{2}{*}{$\max V$} & PLS & & 0.06 & 0.35 & 0.52 & -0.02 & 0.27 & -0.23 & -0.04 & -0.13 & -0.08 & 0.17 \\
\hline & GP & 1.54 & 0.00 & 0.10 & 0.31 & 0.00 & 0.06 & 0.04 & 0.00 & 0.01 & 0.01 & 0.02 \\
\hline \multirow[t]{2}{*}{$\max A$} & PLS & & 0.56 & 0.10 & -0.86 & 0.02 & 0.09 & 0.08 & -0.09 & -0.01 & 0.04 & -0.25 \\
\hline & GP & 2.5 & 0.17 & 0.01 & 0.55 & 0.00 & 0.00 & 0.00 & 0.00 & 0.00 & 0.00 & 0.03 \\
\hline \multirow[t]{2}{*}{$\min 2 \mathrm{~V}$} & PLS & & 0.31 & 0.13 & -0.94 & 0.03 & -0.20 & 0.15 & 0.08 & 0.07 & 0.00 & -0.09 \\
\hline & GP & -0.33 & 0.04 & 0.01 & 0.53 & 0.00 & 0.02 & 0.01 & 0.00 & 0.00 & 0.00 & 0.00 \\
\hline \multirow[t]{2}{*}{$\min 2 \mathrm{~A}$} & PLS & & 0.13 & 0.23 & -0.96 & -0.01 & 0.22 & 0.11 & 0.02 & 0.04 & -0.09 & -0.46 \\
\hline & GP & 45.0 & 0.01 & 0.03 & 0.52 & 0.00 & 0.02 & 0.00 & 0.00 & 0.00 & 0.00 & 0.08 \\
\hline
\end{tabular}




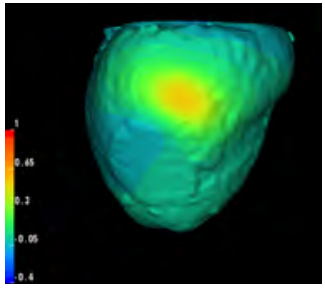

ISC $=\mathbf{2 0 \%}$ : (a) $10-30 \%$

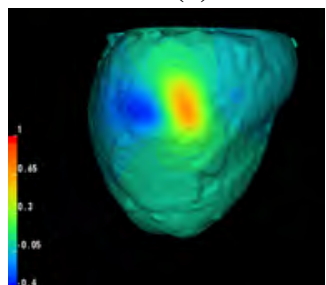

ISC $=\mathbf{2 0 \%}$ : (d) 40-60\%

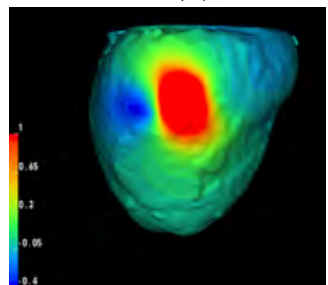

ISCOFF $=70 \%$ : (a) $10-70 \%$

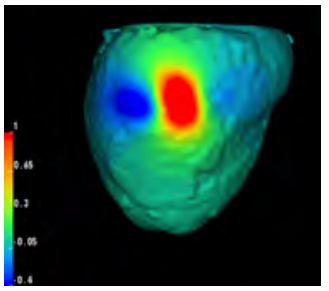

ISCOFF=70\%: (d) $40-70 \%$

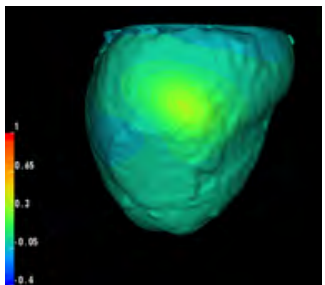

ISCON=20\% : (a) $20-30 \%$

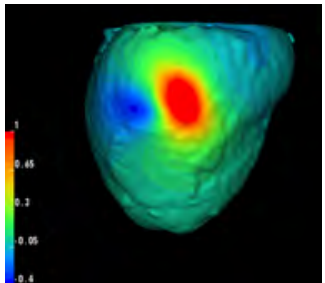

ISCON=20\% : (d) $20-60 \%$

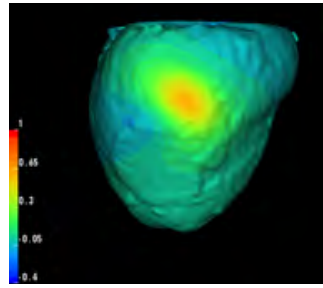

(b) $20-40 \%$

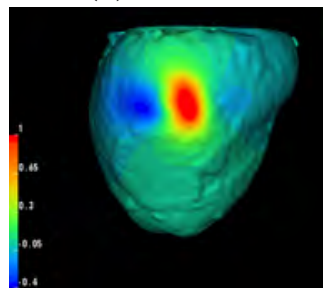

(e) $50-70 \%$

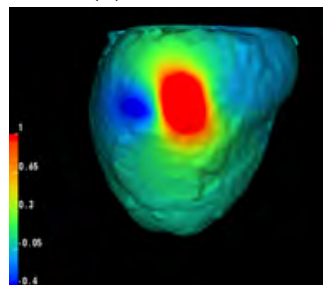

(b) $20-70 \%$

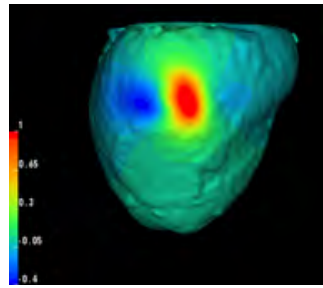

(e) $50-70 \%$

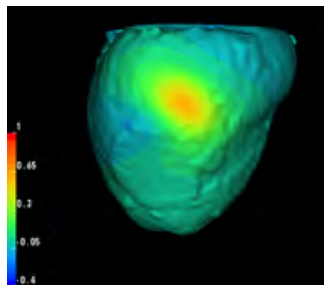

(b) $20-40 \%$

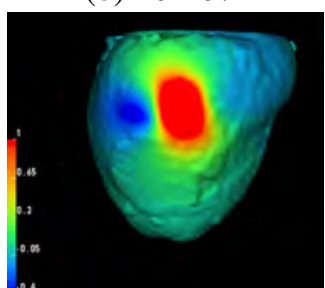

(e) $20-70 \%$

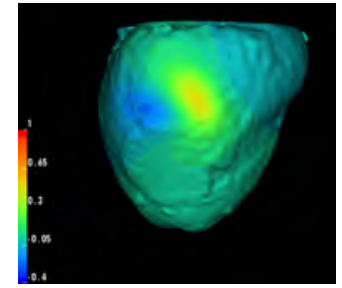

(c) $30-50 \%$

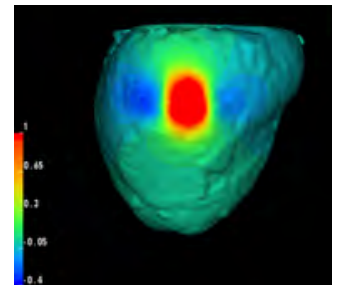

(f) $60-80 \%$

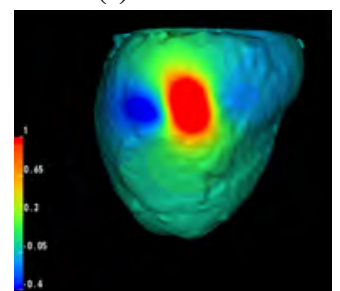

(c) $30-70 \%$

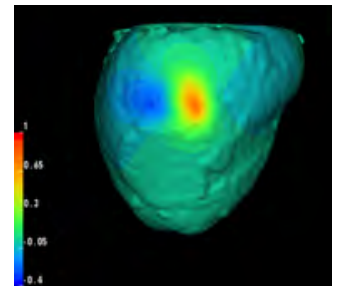

(f) $60-70 \%$

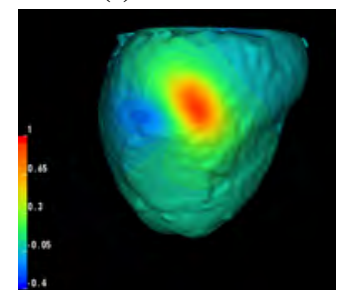

(c) $20-50 \%$

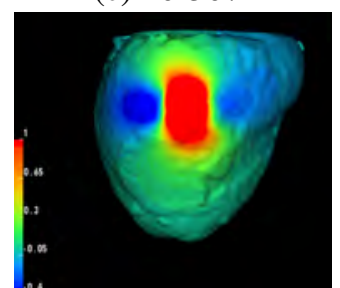

(f) $20-80 \%$

Figure 9: Epicardial potential distributions generated using mean values for the parameters (Table 1) during the Early stage of ischaemia, for the realistic model, for (top two rows) fixed ISCON=20\%, (middle two rows) fixed ISCOFF $=70 \%$, and (bottom two rows) fixed ISCON=20\%, for a range of ischaemic depths, labelled ISCON-ISCOFF (e.g. 20-40\%). Scale is in mV. Dashed contour lines are negative potentials, solid lines positive potentials, and contours range from -0.3 to 0 in steps of $0.05 \mathrm{mV}$. 
$\max \mathrm{V}$ is sensitive to both ISC and ISCOFF, with ISCOFF having the stronger effect. In the case of $\min 1 \mathrm{~V}$, the results indicate a stronger sensitivity to ISC than to $g_{e l}$. We can also see from the sign of the correlation in the PLS coefficients that in each case an increase in the input leads to an increase in the magnitude of the output (positive for $\max \mathrm{V}$ and negative for $\min 1 \mathrm{~V}$ and $\min 2 \mathrm{~V}$ ).

The results in Table 6 also show that the angles maxA and min2A are strongly sensitive to ISCOFF (moving clockwise with increasing ISCOFF) and that min2A is also sensitive to $g_{e n}$, as is min1A. The final input to which both min1A and maxA are sensitive is ROT, and in each case increasing ROT leads to an anticlockwise movement of the feature. So, in summary, increases in ROT and $g_{\text {en }}$ have an anticlockwise effect on min1A, increases in ROT and ISCOFF have opposite effects on maxA (with ISCOFF stronger), and increases in ISCOFF and $g_{e n}$ have a clockwise effect on $\min 2 \mathrm{~A}$.

We can also conclude from this work that none of the outputs is sensitive to $g_{b}, g_{i l}, g_{i t}, g_{e t}$ or $g_{i n}$. Another point to note is that the sums of the values for the GP indices are in the range (0.51-0.78), which is considerably less than 1 . This may indicate a poor fit for the emulators, or, as is likely in this case, that there is a joint effect between two (or more) of the indices on the output [23]. For example, while there is not a direct relationship between ISC and ISCOFF, as ISCON and ISC were both chosen randomly within their ranges, it seems likely, based on the results of Section 3.1, that these two inputs could jointly have an effect on the outputs.

\subsubsection{PTI main effect plots for Early Ischaemia}

To help visualise and quantify the effects of the inputs on the various outputs in the Early ischaemia case, we produced the main effect plots that are given in Figure 10. In each of the plots, a value of 0 on the vertical axis corresponds to the emulator expected mean $E^{*} E\{f(x)\}$ for that output, which can be found in Table 6 . Initial study of the plots shows that not only do they agree with Table 6 , but that they provide extra information about the effects of the inputs on an output. For example, the plot in Figure 10(b) for maxV, indicates that varying ISCOFF across the range considered (15-85\%) can change the value for maxV from approximately $1.54-1.3=0.24 \mathrm{mV}$ to $1.54+1.3=2.84 \mathrm{mV}$, and the value for maxA clockwise from approximately $2.5+6=8.5^{\circ}$ to $2.5-6=-4.5^{\circ}$, assuming that the other inputs are held at their mean values.

Noting the very different scales for the main effect plots for the potentials (Figures 10(a)-(c)), we see that ISCOFF has a much greater effect on maxV and min2V than ISC has on min $1 \mathrm{~V}$. However, this is not the case for the plots for the angles (Figures 10(d)-(f)), where ROT has a greater effect on min1A than on maxA, although it is much less than the effect of ISCOFF on min2A. We can also see the opposite effects of ROT and ISCOFF on maxA.

\subsubsection{PTI sensitivities in other cases}

We repeated the above PTI studies (Sections 3.3.1 and 3.3.2) both for the Late ischaemia scenario and for the scenario where the conductivities were not reduced in the ischaemic region (i.e. the conductivities were the same in and outside of the ischaemic region). The results indicated that the outputs were sensitive to exactly the same inputs as in the Early ischaemia scenario. In addition, the PLS regression coefficients and the GP sensitivity indices had values that were very similar to those in Table 6 .

The emulator expected values $E^{*} E\{f(x)\}$ were lower for the potentials for the Late case than for the Early case, which is consistent with previous work for the SI model [27]. Interestingly, the values for $E^{*} E\{f(x)\}$ for the angles $\min 1 \mathrm{~A}, \max \mathrm{A}$ and $\min 2 \mathrm{~A}$, were quite similar for the Early and Late ischaemia scenarios for the PTI model, indicating that the reductions in the intracellular conductivities in the ischaemic region for the Late scenario had an effect on the potentials of the features but not on their positions.

We also repeated the studies of Sections 3.3.1 and 3.3.2 using the new representation for the transmembrane potential (Equation (6)), for both Early and Late ischaemia, and again found that the outputs were sensitive to the same inputs as previously. Some of these studies had sensitivity values that were border-line as being significant, so we dealt with this by bolding a value in Table 6 only if it was at least moderately sensitive (PLS absolute value greater than 0.3 ) for all studies.

We also performed Early and Late case PTI studies, where we tested the sensitivity of the outputs to the same inputs as above, except that we used ISCON and ISCOFF, instead of ISC and ISCOFF. These studies showed that the outputs were not sensitive to ISCON. This was possibly due to the fact that ISCON was varied across only a small range (0-30\%) in these studies; when we repeated the study with ISCON varying over $0-50 \%$, we found similar results for ISCON as for ISC in Table 6, that is, sensitivity of $\min 1 \mathrm{~V}$ to ISCON.

\subsubsection{SI sensitivities for Early Ischaemia}

We now present work from a study, similar to that in Sections 3.3.2 and 3.3.1, but using the SI model, so that we can compare the PTI and SI models. The SI study was undertaken as part of our previously published paper [27], but it was not presented in detail, as that paper was primarily focused on the effect of changing the conductivities in the ischaemic region. Here we present main effect plots for the SI model for Early ischaemia (Figure 11) to compare with the main effect plots in Figure 10. These SI plots are for type 3 EPDs only, to match those of the PTI case.

If we compare Figure 10 with Figure 11, for each of the six outputs, it is apparent that plots (d) for min2A 


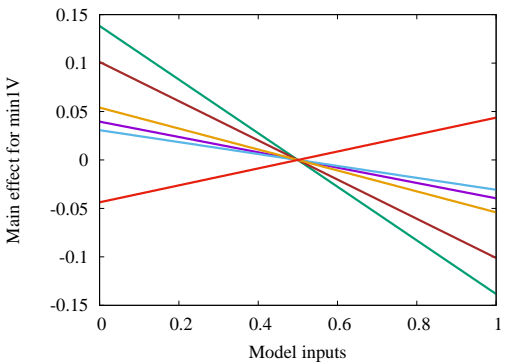

(a) $\min 1 \mathrm{~V}$

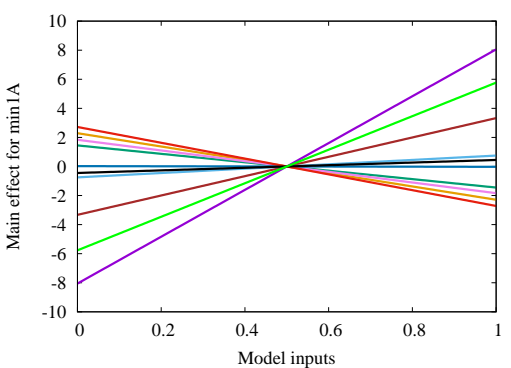

(d) $\min 1 \mathrm{~A}$

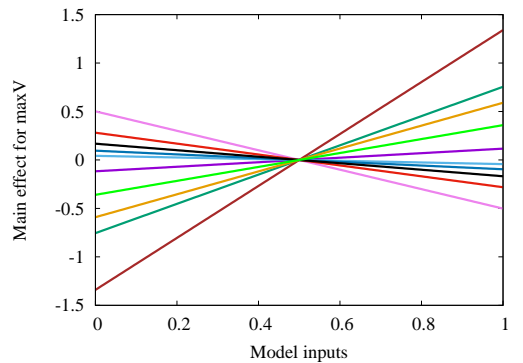

(b) $\max \mathrm{V}$

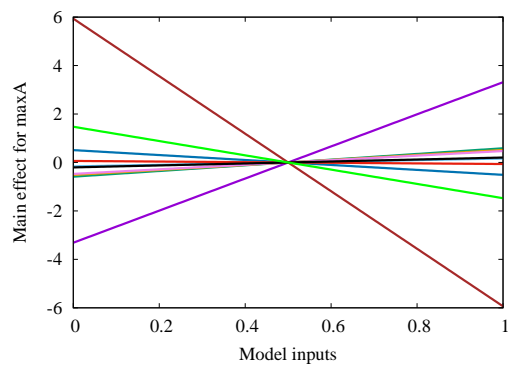

(e) $\max \mathrm{A}$

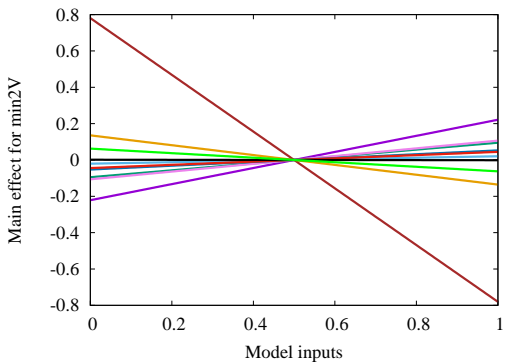

(c) $\min 2 \mathrm{~V}$

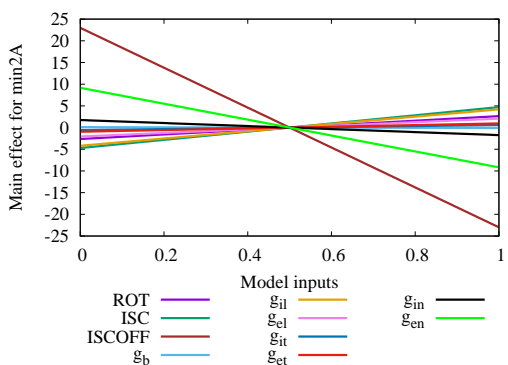

(f) $\min 2 \mathrm{~A}$

Figure 10: Main effect plots for Early stage partial thickness ischaemia. Legend in (f) applies to all plots.

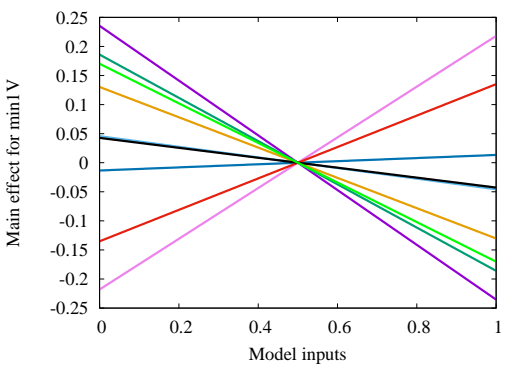

(a) $\min 1 \mathrm{~V}$

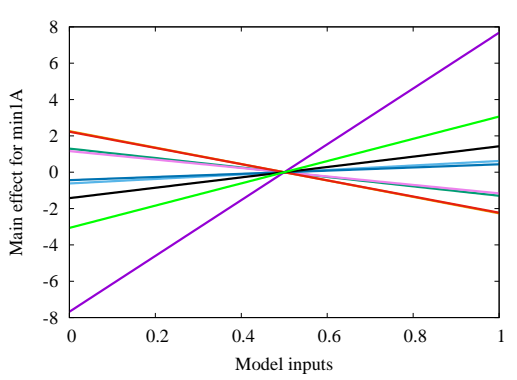

(d) $\min 1 \mathrm{~A}$

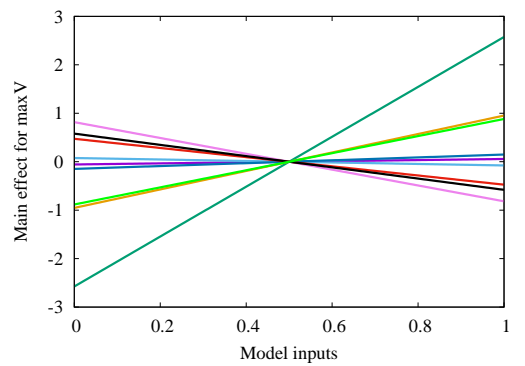

(b) $\max \mathrm{V}$

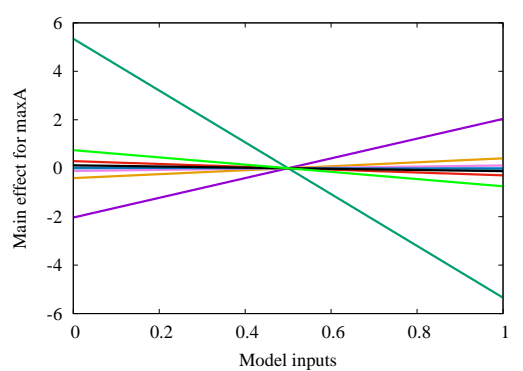

(e) $\max \mathrm{A}$

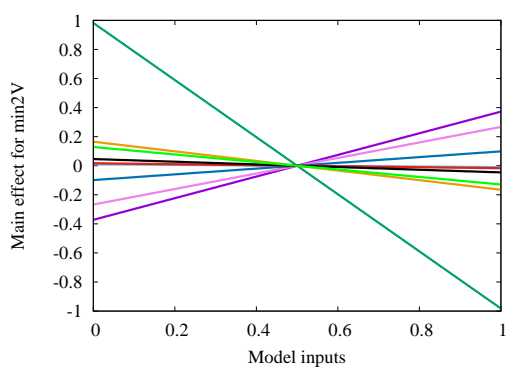

(c) $\min 2 \mathrm{~V}$

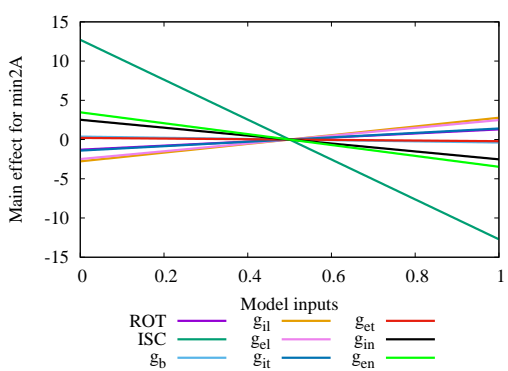

(f) $\min 2 \mathrm{~A}$

Figure 11: Main effect plots for Early stage subendocardial ischaemia (type 3). Legend in (f) applies to all plots. 
Table 7: Emulator expected means $E^{*} E\{f(x)\}$ (in $\mathrm{mV}$ for potentials and ${ }^{\circ}$ for angles) and sensitivities of the EPD type 3 feature outputs in the SI half-ellipsoidal model to nine inputs (see Table 1 and text), for Early stage ischaemia, calculated as GP emulator sensitivities (GP) or by partial least squares (PLS). PLS sensitivities are signed, GP sensitivities are not. Values in bold indicate those inputs to which the output is particularly sensitive.

\begin{tabular}{ll|c|ccccccccc}
\hline Feature & Method & $E^{*} E\{f(x)\}$ & ROT & ISC & $g_{b}$ & $g_{i l}$ & $g_{\text {el }}$ & $g_{i t}$ & $g_{\text {et }}$ & $g_{\text {in }}$ & $g_{\text {en }}$ \\
\hline min1V & PLS & & $\mathbf{- 0 . 5 2}$ & $\mathbf{- 0 . 4 1}$ & -0.10 & -0.29 & $\mathbf{0 . 4 8}$ & 0.03 & 0.30 & -0.09 & -0.38 \\
& GP & -0.77 & $\mathbf{0 . 2 0}$ & $\mathbf{0 . 1 2}$ & 0.01 & 0.06 & $\mathbf{0 . 1 7}$ & 0.00 & 0.07 & 0.01 & 0.10 \\
$\min 1 \mathrm{~A}$ & PLS & & $\mathbf{0 . 7 8}$ & -0.13 & 0.06 & -0.23 & -0.12 & 0.04 & -0.22 & 0.14 & 0.31 \\
& GP & -31.7 & $\mathbf{0 . 4 3}$ & 0.01 & 0.00 & 0.04 & 0.01 & 0.00 & 0.04 & 0.02 & 0.07 \\
\hline $\operatorname{maxV}$ & PLS & & 0.02 & $\mathbf{0 . 8 0}$ & -0.02 & 0.30 & -0.26 & 0.05 & -0.15 & -0.18 & 0.28 \\
& GP & 2.82 & 0.00 & $\mathbf{0 . 4 9}$ & 0.00 & 0.07 & 0.05 & 0.00 & 0.02 & 0.03 & 0.06 \\
$\operatorname{maxA}$ & PLS & & $\mathbf{0 . 3 3}$ & $\mathbf{- 0 . 8 7}$ & 0.00 & 0.07 & 0.02 & 0.00 & -0.05 & -0.02 & -0.12 \\
& GP & 0.22 & $\mathbf{0 . 0 8}$ & $\mathbf{0 . 5 6}$ & 0.00 & 0.00 & 0.00 & 0.00 & 0.00 & 0.00 & 0.01 \\
\hline $\min 2 \mathrm{~V}$ & PLS & & 0.31 & $\mathbf{- 0 . 8 2}$ & -0.01 & -0.14 & 0.22 & 0.08 & -0.01 & -0.04 & -0.11 \\
& GP & -0.78 & 0.07 & $\mathbf{0 . 4 6}$ & 0.00 & 0.01 & 0.03 & 0.01 & 0.00 & 0.00 & 0.01 \\
$\min 2 \mathrm{~A}$ & PLS & & 0.09 & $\mathbf{- 0 . 8 7}$ & -0.03 & 0.19 & 0.17 & 0.10 & -0.01 & -0.17 & -0.24 \\
& GP & \multirow{2}{*}{37.5} & 0.01 & $\mathbf{0 . 5 4}$ & 0.00 & 0.03 & 0.02 & 0.01 & 0.00 & 0.02 & 0.04
\end{tabular}

match qualitatively, and four of the remaining five plots (excluding (a)) are similar to their counterpart, provided that we replace ISC in Figure 11 with ISCOFF in Figure 10. The final plots (a) show similar trends for most inputs, except that the greatest sensitivity is to ISC for the PTI model, whereas it is to ROT for the SI model.

In addition to the plots, we have also tabulated (Table 7) the SI model GP and PLS sensitivities that match the above plots, so that they can be compared with the data in Table 6. The SI model values are for the type 3 EPDs that come from varying ISC in the range 40-90\%, whereas the PTI values come from varying ISC in the range 5-55\% and also varying ISCON, so that ISCOFF varies between 15-85\% (Section 3.3.1). Due to these differences, we would not expect the $E^{*} E\{f(x)\}$ values to be the same; in fact, we see that the magnitude of the expected PTI potentials is much lower in each case than the SI ones, and the angles are also smaller. It is also interesting to note that in the SI case the $E^{*} E\{f(x)\}$ values for min1V and min2V are nearly the same, whereas in the PTI case the magnitude of $\min 2 \mathrm{~V}$ is larger than min $1 \mathrm{~V}$.

\subsubsection{Sensitivity comparison for SI and PTI models}

We now summarise the results from Tables 6 and 7 in Table 8(a), where a tick indicates that an output is sensitive to that particular input. We see that min $1 \mathrm{~V}$ is sensitive to ROT, ISC and $g_{e l}$ in the SI model, but only to ISC and $g_{e l}$ in the PTI model, and that in both models min1A is most sensitive to ROT. In addition, we note in the SI case all max and min2 outputs are most sensitive to ISC, whereas for the PTI model they are most sensitive to ISCOFF. As well as sensitivity to ROT and ISC(ISCOFF), in some cases there are weaker sensitivities to other inputs. These do not always match between the two models: sensitivity of min1V to $g_{e l}$ appears in both models, whereas significant sensitivity of $\min 1 \mathrm{~A}$ and $\min 2 \mathrm{~A}$ to $g_{\text {en }}$, and
maxA to ROT, appears in the PTI model, but not the SI model where the sensitivity is weaker.

Since ISC in the SI model is the same as ISCOFF (as ISCON $=0 \%$, we replace ISC with ISCOFF in Table 8(a) for the SI model and combine these into one column in Table 8(b) (red ticks). The only case where we do not do this is for min $1 \mathrm{~V}$ as the significant input for the PTI model is ISC in this case, so we leave ISC for SI to match it. This change means that, for all four max and min2 features, the two models agree that it is ISCOFF and not ISC that is the significant input.

\section{Discussion}

Recent experimental results have challenged the commonly held position that ischaemia develops initially at the endocardium, and instead suggested that in many cases ischaemia develops in the mid-wall [11]. This has provided the motivation for this study, which compares the EPDs that are produced under the conditions of subendocardial and partial thickness ischaemia. For the PTI studies, it is necessary to consider not just ISC, as in previous studies $[25,27]$, but also ISCON and ISCOFF.

Initial studies using mean input parameters (Table 1), where we considered various combinations of ISC, ISCON and ISCOFF, showed that there were both similarities and differences between the EPDs produced by the two models. One major difference was that the PTI model generally only produced type 3 EPDs (that is, EPDs that have a maximum over the ischaemic region, flanked by two minima), unlike the SI model where all three types of EPDs can be found [27].

This is consistent with conclusions that can be drawn from the model of extracellular current pathways, in and around the ischaemic zone, which was proposed by Hopenfeld et al. [28]. The model is based on the fact that the 


\begin{tabular}{llccccc} 
Output & Model & ROT & ISC & ISCOFF & $g_{e l}$ & $g_{e n}$ \\
\hline $\min 1 \mathrm{~V}$ & SI & $\checkmark$ & $\checkmark$ & & $\checkmark$ & \\
& PTI & & $\checkmark$ & & $\checkmark$ & \\
\hline $\min 1 \mathrm{~A}$ & SI & $\checkmark$ & & & & \\
& PTI & $\checkmark$ & & & & $\checkmark$ \\
\hline $\max \mathrm{V}$ & SI & & $\checkmark$ & & & \\
& PTI & & & $\checkmark$ & & \\
\hline $\max \mathrm{A}$ & SI & $\checkmark$ & $\checkmark$ & & & \\
& PTI & $\checkmark$ & & $\checkmark$ & & \\
\hline $\min 2 \mathrm{~V}$ & SI & & $\checkmark$ & & & \\
& PTI & & & $\checkmark$ & & \\
\hline $\min 2 \mathrm{~A}$ & SI & & $\checkmark$ & & & \\
& PTI & & & $\checkmark$ & & $\checkmark$
\end{tabular}

(a) All sensitive inputs

\begin{tabular}{llcccc} 
Output & Model & ROT & ISCOFF & $g_{e l}$ & $g_{e n}$ \\
\hline $\min 1 \mathrm{~V}$ & SI & $\checkmark$ & ISC & $\checkmark$ & \\
& PTI & & ISC & $\checkmark$ & \\
\hline $\min 1 \mathrm{~A}$ & SI & $\checkmark$ & & & \\
& PTI & $\checkmark$ & & & $\checkmark$ \\
\hline $\operatorname{maxV}$ & SI & & $\checkmark$ & & \\
& PTI & & $\checkmark$ & & \\
\hline $\operatorname{maxA}$ & SI & $\checkmark$ & $\checkmark$ & & \\
& PTI & $\checkmark$ & $\checkmark$ & & \\
\hline $\min 2 \mathrm{~V}$ & SI & & $\checkmark$ & & \\
& PTI & & $\checkmark$ & & \\
\hline $\min 2 \mathrm{~A}$ & SI & & $\checkmark$ & & \\
& PTI & & $\checkmark$ & & $\checkmark$
\end{tabular}

(b) All sensitive inputs (ISC $\rightarrow$ ISCOFF)

Table 8: A comparison of sensitivity of outputs to inputs in the SI and PTI models, with (a) sensitive inputs, and (b) sensitive inputs, with SI ISC relabelled ISCOFF (red ticks).

strength of the extracellular voltage drop, over the lateral and transmural boundaries of the ischaemic zone, varies depending on the thickness of the ischaemic region and the orientation of the cardiac fibres to the boundaries. For the ischaemic region shape used here, and given that, for the PTI model both the transmural boundaries are in contact with healthy tissue (and not blood at the endocardium as in the SI model), then the model [28] suggests that the voltage drop over the lateral boundaries is greater than that over the transmural boundaries. This always leads to a maximum over the ischaemic region in the PTI model, unlike the SI model where it is possible to see a minimum over the ischaemic region.

Sensitivity analysis and uncertainty quantification studies were performed (Section 3.3) to try to tease out the effects of ISC, ISCON and ISCOFF on the form of the EPDs. These sensitivity analyses were then compared with a similar study for the SI model (Section 3.3.4). A major finding from this comparison was that the four EPD outputs associated with $\max$ and $\min 2(\max V, \max \mathrm{A}, \min 2 \mathrm{~V}, \min 2 \mathrm{~A})$ were sensitive to ISCOFF in both models, rather than to ISC. This is consistent with the lack of type 1 and 2 EPDs in the PTI model, since low ISC (generally less than $30 \%$ ) was previously found $[4,5,25,27]$ to be associated with these EPDs in the SI model, and so we would not expect to see features such as min 2 when ISCOFF values are low. An analysis of the eight type 2 EPDs from Section 3.3 , found ISCOFF in the range $19-32 \%$, with ISC in the range $6-20 \%$, which is consistent with this hypothesis.

The rarity of types 1 and 2 EPDs in PTI simulations could be significant from a clinical point of view. We previously suggested [25] that the appearance of a second min- imum (min2) might be one way of determining whether or not SI is significant clinically, since min2 generally does not appear for SI until ISC is greater than about $30-40 \%$. However, since we have found that types 1 and 2 EPDs are rare in PTI simulations, then, if this corresponds to what is seen clinically, this method no longer seems feasible.

Allowing for the change of SI ISC to ISCOFF, the sensitivity study comparison between the SI and PTI models showed that the outputs were generally sensitive/not sensitive to the same inputs. For example, neither model showed sensitivity to $g_{b}, g_{\text {et }}$ or any of the intracellular conductivities, whereas both models showed sensitivity to ROT, ISCOFF and/or ISC, and $g_{e l}$. The main differences were in the lack of sensitivity to ROT for PTI min1V, and the sensitivities to $g_{e n}$ for min1A and min2A, which were stronger in the PTI model than in the SI model.

One other result to consider is the fact that max and $\min 2$ are sensitive to ISCOFF, whereas min $1 \mathrm{~V}$ is sensitive to ISC. Since ISCOFF does not indicate the degree of ischaemia as ISC does, it may be more useful to focus on min1 when trying to characterise ischaemia via the ECG. However, the sensitivity of min1A (i.e. the position of min1) to ROT also needs to be taken in account, given the possible variation of ROT in the general population [42]. This might mean that min1 could present in different positions on the heart surface, and perhaps even the torso surface, for different patients, with resultant effects on the ECG.

We have also shown that it makes no difference to which inputs are identified as being those to which the outputs are most sensitive, when one simulates either Early or Late stage ischaemia, and that this is the case for both the 
PTI and SI models. However, in both models, the magnitudes of the potentials are less in the Late case than in the Early case, which may be significant clinically as features such as the minima may be more difficult to detect.

This work has shown that it is possible for models of non-transmural ischaemia to produce EPDs of types 1, 2 and 3 , but it would appear that type 1 EPDs (a single minimum over the ischaemic region) only occur for SI (ISCON=0\%) with low ISCOFF. Similarly, type 2 EPDs (max, plus one min) can occur for low ISCON (not necessarily $0 \%$ ) coupled with low ISCOFF. In modelling, both types 1 and 2 EPDs are rare in comparison with type 3 EPDs (a maximum plus two minima), and this is consistent with experimental results, such as those found by Aras et al. [11], both in terms of the EPD patterns found, and in terms of the positions where ischaemia arises (i.e. only in about $10 \%$ of cases is it subendocardial).

Recent experimental work [15] has shown that subepicardial ischaemic zones produced elevated heart surface potentials, and zones that were located in a more subendocardial position tended to produce weaker elevations. This is consistent with our finding that $\operatorname{maxV}$ is sensitive to ISCOFF (with a positive correlation). Our finding that the position and magnitude of $\max$ and $\min 2$ are sensitive to the proximity of the ischaemic region to the epicardium (i.e. ISCOFF) rather than to the ischaemic depth is a novel result of this study. It is not inconsistent with previous experimental and modelling work (conducted under the assumption that the transverse and normal conductivities are equal) $[4,5,25,27,28,29,30,43,44]$. Those studies highlighted the connection between increasing ischaemic depth and increasing magnitude of the epicardial maxima and minima (and clockwise rotation of their position), and this matches our work because, in SI modelling, ISC and ISCOFF are the same. However, our work does indicate a different relationship when one considers ISCOFF rather than ISC, especially in PTI modelling.

This study has focused on comparing two similar models of non-transmural ischaemia, and then discussed the implications of the modelling for EPD patterns and what this might mean for the ECG, if the results were to translate to the torso surface. Apart from this, there are various other limitations to the modelling, which is restricted to the left ventricle, under the assumption that the walls have uniform thickness. The modelling also makes certain assumptions about the form of the transmembrane potential and the ischaemic zone. Our work has found similar results using two different representations for the transmembrane potential, for both models. The choice here of a single rectangularly-shaped ischaemic zone is another limitation, although previous work [12] has shown that a cylindrical ischaemic zone does produce similar EPDs. We have also made assumptions about the size and position of the ischaemic zone. However, our previous work [25] has found that, with ischaemic zones up to twice the area as used here, the size of the ischaemic zone affects the magnitude of the potentials, but it does not affect the particular in- puts to which the outputs are the most sensitive. This is also the case when the position of the ischaemic zone is changed, provided it is not located near the apex or base of the ventricle [25]. Since ventricular contraction begins during the ST segment of the ECG, a further limitation of this model relates to the assumption, made here, that the blood volume is unchanged throughout the ST segment, given that a recent modelling [45] has shown that a reduction in blood volume affects ST depression.

Future work might look at using more than one ischaemic zone, but this would introduce extra input variables and it may be difficult to characterise the interaction between the two zones. In addition, a recent study [15] has found that using a single ischaemic zone produced more realistic (i.e. matching measured potentials) ST potential distributions than using their previous 'needle-based' source potential model. Other future work could include using a PTI model in a realistic heart geometry that is coupled with a torso model, as well as including a cell model to overcome the limitation associated with this work of using the passive bidomain model.

\section{Conclusion}

This study has presented a comparison of an SI model and a PTI model for studying the ST segment EPD features (maximum and minima) that are generated by the differences in transmembrane potential between the ischaemic and normal tissue. The comparison of the two models was motivated by an experimental study [11] that found that ischaemia is more likely to arise within the ventricular wall than at the endocardium.

We found that both models have no sensitivity to the blood conductivity and the intracellular and transverse bidomain conductivities. In addition, we determined that the most important inputs are ROT, ISC (for min1V) and ISCOFF (for potentials and positions of min2 and max), and that type 1 EPDs (a single minimum over the ischaemic region) occur only in the SI model with low ISC, and not in the PTI model. In order to ensure that modelling reflects the above experimental results, but also allows type 1 EPDs to occur, we suggest that studies use a PTI model with ISCON varying from $0 \%$ upwards (preferably with $\mathrm{ISCON}=0 \%$ chosen to occur in around $10 \%$ of simulations, as per the experimental results [11]).

\section{References}

[1] R. H. Clayton, O. Bernus, E. M. Cherry, H. Dierckx, F. H. Fenton, L. Mirabella, A. V. Panfilov, F. B. Sachse, G. Seemann, H. Zhang, Models of cardiac tissue electrophysiology: Progress, challenges and open questions, Progress in Biophysics and Molecular Biology 104 (1-3) (2011) 22-48.

[2] B. Rodriguez, N. Trayanova, D. Noble, Modelling cardiac ischaemia, Ann. N.Y. Acad. Sci. 1080 (2006) 395-414.

[3] P. Colli Franzone, L. F. Pavarino, S. Scacchi, Dynamical effects of myocardial ischemia in anisotropic cardiac models in three dimensions, Mathematical Models and Methods in Applied Sciences 17 (12) (2007) 1965-2008. 
[4] B. Hopenfeld, J. G. Stinstra, R. S. MacLeod, The effect of conductivity on ST-segment epicardial potentials arising from subendocardial ischemia, Annals of Biomedical Engineering 33 (6) (2005) 751-763.

[5] M. Potse, R. Coronel, S. Falcao, A. R. LeBlanc, A. Vinet, The effect of lesion size and tissue remodeling on ST deviation in partial-thickness ischemia;, Heart Rhythm 4 (2) (2007) 200-206.

[6] G. T. Lines, B. L. de Oliveira, O. Skavhaug, M. M. Maleckar, Simple T-wave metrics may better predict early ischaemia as compared to ST segment, IEEE Transactions on Biomedial Engineering 64 (6) (2017) 1305-1309.

[7] K. Akkerhuis, M. Simoons, Comprehensive electrocardiology, Springer-Verlag, London, 2011, Ch. 36. Exercise electrocardiography and exercise testing, pp. 1677-1719.

[8] R. L. Wilensky, J. Tranum-Jensen, R. Coronel, A. A. M. Wilde, J. T. Fiolet, M. J. Janse, The subendocardial border zone during acute ischemia of the rabbit heart: an electrophysiologic, metabolic, and morphologic correlative study, Circulation 74 (5) (1986) 1137-1146.

[9] M. J. Janse, A. G. Kleber, A. Capucci, R. Coronel, F. WilmsSchopman, Electrophysical basis for arrythmia caused by acute ischemia, Journal of Molecular and Cellular Cardiology 18 (1986) 339-355.

[10] R. Coronel, J. W. T. Fiolet, F. J. G. Wilms-Schopman, A. F. M. Schaapherder, T. A. Johnson, L. S. Gettes, M. J. Janse, Distribution of extracelllular potassium and its relation to electrophysiologic changes during acute myocardial ischemia in the isolated perfused porcine heart, Circulation 77 (5) (1988) 11251138 .

[11] K. Aras, B. Burton, D. Swenson, R. MacLeod, Spatial organisation of acute myocardial ischaemia, Journal of Electrocardiology 49 (2016) 323-336

[12] J. P. Barnes, P. R. Johnston, How ischaemic region shape affects ST potentials in models of cardiac ischaemia, Mathematical Biosciences 239 (213-221) (2012).

[13] B. M. Johnston, S. Coveney, E. T. Y. Chang, P. R. Johnston, R. H. Clayton, Quantifying the effect of uncertainty in input parameters in a simplified bidomain model of partial thickness ischaemia, Medical and Biological Engineering and Computing 56 (2018) 761-780.

[14] P. R. Johnston, D. Kilpatrick, The effect of conductivity values on ST segment shift in subendocardial ischaemia, IEEE Transactions on Biomedical Engineering 50 (2) (2003) 150-158.

15] B. M. Burton, K. K. Aras, W. W. Good, J. D. Tate, B. Zenger, R. S. MacLeod, Image-based modeling of acute myocardial ischaemia using experimentally derived ischemic source zone representations, Journal of Electrocardiology 51 (2018) 725-733.

[16] A. Muszkiewicz, O. J. Britton, P. Gemmell, E. Passini, C. Sánchez, X. Zhou, A. Carusi, T. A. Quinn, K. Burrage, A. Bueno-Orovio, B. Rodriguez, Variability in cardiac electrophysiology: Using experimentally-calibrated populations of models to move beyond the single virtual physiological human paradigm, Progress and Biophysics and Molecular Biology $120(1-3)(2016) 115-127$.

[17] S. E. Geneser, R. M. Kirby, R. S. MacLeod, Application of stochastic finite element methods to study the sensitivity of ECG forward modeling to organ conductivity, IEEE Transactions in Biomedical Engineering 55 (1) (2008) 31-40.

[18] Z. Hu, D. Du, Y. Du, Generalized polynomial chaos-based uncertainty quantification and propagation in multi-scale modeling of cardiac electrophysiology, Computers in Biology and Medicine 102 (2018) 57-74.

[19] P. R. Johnston, Defibrillation thresholds: A generalised polynomial chaos study, in: A. Murray (Ed.), Computing in Cardiology, Vol. 41, IEEE, IEEE Press, 2014, pp. 197-200.

[20] J. Dhamala, H. J. Arevalo, J. Sapp, B. M. Horácek, K. C. Wu, N. A.Trayanova, L. Wang, Quantifying the uncertainty in model parameters using Gaussian Process-based Markov chain Monte Carlo in cardiac electrophysiology, Journal of Medical Image Analysis (2018) DOI: 10.1016/j.media.2018.05.007.

[21] R. H. Johnstone, E. T. Y. Chang, R. Bardenet, T. P. de Boer,
D. J. Gavaghan, P. Pathmanathan, R. H. Clayton, G. R. Mirams, Uncertainty and variability in models of the cardiac action potential: Can we build trustworthy models?, Journal of Molecular and Cellular Cardiology 96 (2016) 49-62.

[22] J. E. Oakley, A. O'Hagan, Probabilistic sensitivity analysis of complex models: a Bayesian approach, Journal of the Royal Statistical Society: Series B (Statistical Methodology) 66 (751769) (2004)

[23] E. T. Y. Chang, M. Strong, R. H. Clayton, Bayesian sensitivity analysis of a cardiac cell model using a Gaussian Process emulator, PLoS ONE 10 (6) (2015) e0130252.

[24] S. Coveney, R. H. Clayton, Fitting two human atrial cell models to experimental data using bayesian history matching, Progress in Biophysics and Molecular Biology (2018) DOI: 10.1016/j.pbiomolbio.2018.08.001.

[25] B. M. Johnston, P. R. Johnston, Determining the most significant input parameters in models of subendocardial ischaemia and their effect on ST segment epicardial potential distributions, Computers in Biology and Medicine 95 (2018) 75-89.

[26] B. A. Lawson, K. Burrage, P. Burrage, C. C. Dovandi, A. Bueno-Orovio, Slow recovery of excitability increases ventricular fibrillation risk as identified by emulation, Frontiers in Physiology 9 (1114) (2018) 1-19.

[27] B. M. Johnston, P. R. Johnston, Sensitivity analysis of STsegment epicardial potentials arising from changes in ischaemic region conductivities in early and late stage ischaemia, Computers in Biology and Medicine 102 (2018) 288-299.

[28] B. Hopenfeld, J. G. Stinstra, R. S. MacLeod, Mechanism for ST depression associated with contiguous subendocardial ischaemia, Journal of Cardiovascular Electrophysiology 15 (2004) $1200-1206$.

[29] S. Shome, J. G. Stinstra, B. Hopenfeld, B. B. Punske, R. S. MacLeod, A study of the dynamics of cardiac ischaemia using experimental and modeling approaches, in: Proceedings of the IEEE Engineering in Medicine and Biology 26th Annual International Conference, IEEE EMBS, IEEE Press, 2004, pp. $3585-3588$.

[30] J. G. Stinstra, B. Hopenfeld, R. S. Macleod, Using models of the passive cardiac conductivity and full heart anisotropic bidomain to study the epdicardial potentials in ischaemia, in: Proceedings of the IEEE Engineering in Medicine and Biology 26th Annual International Conference, IEEE EMBS, IEEE Press, 2004, pp. $3555-3558$

[31] O. H. Schmitt, Biological information processing using the concept of interpenetrating domains, in: K. N. Leibovic (Ed.), Information Processing in the Nervous System, Springer-Verlag, New York, 1969, Ch. 18, pp. 325-331.

[32] L. Tung, A bi-domain model for describing ischaemic myocardial D-C potentials, Ph.D. thesis, Massachusetts Institute of Technology (June 1978).

33] P. R. Johnston, A finite volume method solution for the bidomain equations and their application to modelling cardiac ischaemia, Computer Methods in Biomechanics and Biomedical Engineering 13 (2) (2010) 157-170.

[34] P. Colli Franzone, L. Guerri, Spreading of excitation in 3-D models of the anisotropic cardiac tissue I: Validation of the eikonal model, Mathematical Biosciences 113 (1993) 145-209.

[35] R. Coronel, F. J. G. Wilms-Schopman, T. Opthof, F. J. L. van Capelle, M. J. Janse, Injury current and gradients of diastolic stimulation threshold, TQ potential, and extracellular potassium concentration during acute regional ischemia in the isolated perfused pig heart, Circulation Research 68 (1991) 12411249

[36] M. Potse, R. Coronel, A. R. LeBlanc, A. Vinet, The role of extracellular potassium transport in computer models of the ischemic zone, Medical and Biological Engineering and Computing 45 (12) (2007) 1187-1199.

[37] J. Stinstra, B. Hopenfeld, R. MacLeod, On the passive cardiac conductivity, Annals of Biomedical Engineering 33 (12) (2005) 1743-1751.

[38] J. P. Barnes, Mathematically modeling the electrophysiological 
effects of ischaemia in the heart, Ph.D. thesis, Griffith University, Brisbane, Australia (2013).

[39] H. Abdi, Partial least squares regression (PLS-regression), Encyclopedia for research methods for the social sciences, Sage, 2003, pp. 792-795.

[40] E. Sobie, Parameter sensitivity analysis in electrophysiological models using multivariate regression, Biophysical Journal 96 (2009) 1264-1274.

[41] P. Geladi, B. R. Kowalski, Partial least squares regression: a tutorial, Analytica Chimica Acta 185 (1986) 1-17.

[42] H. Lombaert, J. Peyrat, P. Croisille, S. Rapacchi, L. Fanton, F. Cheriet, P. Clarysse, I. Magnin, H. Delingette, N. Ayache, Human atlas of the cardiac fiber architecture: study on a healthy population, IEEE Transactions on Medical Imaging 31 (7) (2012) 1436-1447.

[43] M. C. MacLachlan, J. Sundnes, G. T. Lines, Simulation of ST segment changes during subendocardial ischemia using a realistic 3-D cardiac geometry, IEEE Transactions on Biomedical Engineering 52 (5) (2005) 799-807.

[44] J. G. Stinstra, S. Shome, B. Hopenfeld, R. S. MacLeod, Modelling passive cardiac conductivity during ischaemia, Medical \& Biological Engineering \& Computing 43 (6) (2005) 776-782.

[45] B. M. Burton, J. D. Tate, W. Good, R. S. MacLeod, The role of reduced left ventricular, systolic blood volumes in ST segment potentials overlying diseased tissue of the ischaemic heart, in: A. Murray (Ed.), Computing in Cardiology, Vol. 43, 2016, pp. 209-212. 